\section{Istituto}

Nazionale

Fisica

Nucleare
Sezione SANITÀ

Istituto Superiore di Sanità

Viale Regina Elena 299

I-00161 Roma, Italy

INFN-ISS 96/1

January 1996

\title{
Calculation of the Isgur-Wise function from a light-front constituent quark model ${ }^{0}$
}

\author{
S. Simula \\ Istituto Nazionale di Fisica Nucleare, Sezione Sanità \\ Viale Regina Elena 299, I-00161 Roma, Italy
}

\begin{abstract}
The space-like elastic form factor of heavy-light pseudoscalar mesons is investigated within a light-front constituent quark model in order to evaluate the Isgur-Wise form factor. The relativistic composition of the constituent quark spins is properly taken into account using the Melosh rotations, and various heavy-meson wave function are considered, including the eigenfunctions of an effective light-front mass operator reproducing meson mass spectra. It is shown that in a wide range of values of the recoil the Isgur-Wise form factor exhibits a moderate dependence upon the choice of the heavy-meson wave function and is mainly governed by the effects of the confinement scale.
\end{abstract}

PACS numbers: 12.39.Ki, 12.39.Pn, 13.40.Gp

a To appear in Physics Letters B (1996). 
Weak decays of hadrons can provide relevant information on the fundamental parameters of the standard model of the electroweak interaction and on the internal structure of hadrons. The extraction of the Cabibbo-Kobayashi-Maskawa [1] matrix elements from the experiments requires therefore a precise knowledge of electroweak hadron form factors. As is well known, in case of hadrons containing a single heavy-quark $Q\left(m_{Q} \gg \Lambda_{Q C D}\right)$ the complexity of the theoretical analysis is strongly reduced by the Heavy Quark Symmetry $(H Q S)$, i.e. a spin-flavour symmetry that is a consequence of $Q C D$ in the limit of infinite quark masses [2, [3]. The $H Q S$ requires that, when $m_{Q} \rightarrow \infty$, all the non-perturbative strong physics describing the weak decays of heavy hadrons is contained in a single universal function, known as the Isgur-Wise $(I W)$ form factor $\xi^{(I W)}(\omega)$, where $\omega \equiv v \cdot v^{\prime}$ and $v_{\mu}\left(v_{\mu}^{\prime}\right)$ is the four-velocity of the initial (final) hadron. However, the $H Q S$ does not help in predicting the $I W$ function itself, so that several attempts 田 have been made to calculate $\xi^{(I W)}(\omega)$ both from the fundamental theory and models, like the quark models of Refs. [0, 6, (7]. In such models a simple gaussian-like ansatz for the heavy-meson wave function has been adopted. Furthermore, the relativistic treatment of the light-quark spin requires a particular care; as a matter of fact, it has been shown [7] that relativistic effects remarkably increase the slope of the $I W$ form factor at the zero-recoil point $\left(\rho^{2} \equiv-\left[d \xi^{(I W)}(\omega) / d \omega\right]_{\omega=1}\right)$.

The aim of this letter is to investigate the $I W$ form factor within a light-front constituent quark $(C Q)$ model, where: i) the relativistic composition of the $C Q$ spins is properly taken into account using the Melosh rotations [8], and ii) various heavy-meson wave function are considered, including the eigenfunctions of a light-front mass operator, constructed from the effective $q \bar{q}$ interaction of Godfrey and Isgur $(G I)$ [9], which nicely fits meson mass spectra. Our $C Q$ model has been already applied to the investigation of the leptonic decay constant of light and heavy pseudoscalar $(P S)$ mesons [10], the electromagnetic (e.m.) form factors of light $P S$ [10] and vector [11] mesons, the nucleon elastic and $N-\Delta$ transition e.m. form factors 12]. In this letter, the $C Q$ model formulated on the light-front is used to investigate the elastic form factor of heavy-light $P S$ mesons at space-like values of the squared four-momentum transfer $q^{2} \equiv q \cdot q \leq 0$ in order to evaluate the $I W$ form factor. The choice of the space-like sector is motivated by the fact that, only for $q^{2} \leq 0$, the contribution of the so-called Z-graph (pair creation from the vacuum) can be suppressed by choosing an appropriate reference frame [13] (viz. a frame in which $q^{+}=q^{0}+\hat{n} \cdot \vec{q}=0$ where the vector $\hat{n}=(0,0,1)$ defines the spin quantization axis). In a wide range of values of the recoil the $I W$ form factor is found to be mainly governed by the effects of the confinement scale and, in particular, it is slightly affected by the high-momentum components generated in the heavy-meson wave function by the one-gluon-exchange $(O G E)$ term of the effective $G I$ interaction.

As is well known (cf. [14), light-front hadron wave functions are eigenfunctions of the mass operator $\mathcal{M}=M_{0}+\mathcal{V}$ and of the non-interacting angular momentum operators $j^{2}$ and $j_{n}$, where $M_{0}$ is the free-mass operator and $\mathcal{V}$ a Poincaré-invariant interaction term. The operator $M_{0}$ reads as $M_{0}^{2}=\frac{k_{\perp}^{2}+m_{q}^{2}}{\xi}+\frac{k_{\perp}^{2}+m_{\bar{q}}^{2}}{1-\xi}$, where $m_{q}\left(m_{\bar{q}}\right)$ is the constituent quark (antiquark) mass and the intrinsic light-front variables are $\vec{k}_{\perp}=\vec{p}_{q \perp}-\xi \vec{P}_{\perp}$ and $\xi=p_{q}^{+} / P^{+}$, where the subscript $\perp$ indicates the projection perpendicular to $\hat{n}$ and the plus component 
of a 4 -vector $p \equiv\left(p^{0}, \vec{p}\right)$ is given by $p^{+}=p^{0}+\hat{n} \cdot \vec{p}$. Finally, $\tilde{P} \equiv\left(P^{+}, \vec{P}_{\perp}\right)=\tilde{p}_{q}+\tilde{p}_{\bar{q}}$ is the light-front meson momentum and $\tilde{p}_{q}$ the $C Q$ one. It should be pointed out that the centre-of-mass motion is exactly factorized out. Moreover, in terms of the longitudinal momentum $k_{n}$, defined as $k_{n}=(\xi-1 / 2) M_{0}+\left(m_{\bar{q}}^{2}-m_{q}^{2}\right) / 2 M_{0}$, the free-mass $M_{0}$ acquires a more familiar form, viz. $M_{0}=\sqrt{m_{q}^{2}+k^{2}}+\sqrt{m_{\bar{q}}^{2}+k^{2}}$, with $k^{2}=k_{\perp}^{2}+k_{n}^{2}$. Omitting for the sake of simplicity the colour degrees of freedom, the requirement of Poincarè covariance for the intrinsic wave function $\chi\left(\xi, \vec{k}_{\perp}, \nu \bar{\nu}\right)$ of a $P S$ meson implies (cf., e.g., [10])

$$
\chi\left(\vec{k}_{\perp}, \xi, \nu \bar{\nu}\right)=\mathcal{R}\left(\vec{k}_{\perp}, \xi, \nu \bar{\nu}\right) w^{P S}\left(k^{2}\right) \sqrt{J\left(\vec{k}_{\perp}, \xi\right)}
$$

where $\nu, \bar{\nu}$ are the $C Q$ spin variables and $J\left(\vec{k}_{\perp}, \xi\right)=\frac{M_{0}}{16 \pi \xi(1-\xi)}\left[1-\left(\frac{m_{q}^{2}-m_{\bar{q}}^{2}}{M_{0}^{2}}\right)^{2}\right]$ is the Jacobian of the transformation between $k_{n}$ and $\xi$. In Eq. (1) the momentum-dependent quantity $\mathcal{R}$ is a unitary matrix arising from the Melosh rotations of the $C Q$ spins; its explicit expression can be found, e.g., in [15].

The wave function $w^{P S}\left(k^{2}\right)|00\rangle$, where $|00\rangle=\sum_{\nu \bar{\nu}}\left\langle\frac{1}{2} \nu \frac{1}{2} \bar{\nu} \mid 00\right\rangle\left|\frac{1}{2} \nu\right\rangle\left|\frac{1}{2} \bar{\nu}\right\rangle$ is the canonical quark-spin wave function, is eigenfunction of the transformed mass operator $M=\mathcal{R} \mathcal{M} \mathcal{R}^{\dagger}=$ $\mathcal{R} M_{0} \mathcal{R}^{\dagger}+\mathcal{R} \mathcal{V} \mathcal{R}^{\dagger}$. Since the free-mass commutes with the Melosh rotation, one has $M=$ $M_{0}+V$, where the interaction $V=\mathcal{R} \mathcal{V} \mathcal{R}^{\dagger}$ has to be invariant upon rotations and independent of the total momentum and the centre-of-mass coordinates (cf. [14]). Following Refs. [10, 11], the Melosh-rotated mass operator $M$ is chosen to be the effective Hamiltonian, $H_{q \bar{q}}$, proposed in [9] for the description of meson mass spectra. Therefore, the radial wave function $w^{P S}\left(k^{2}\right)$, appearing in Eq. (1), is eigenfunction of the effective GI Hamiltonian, viz.

$$
H_{q \bar{q}} w^{P S}\left(k^{2}\right)|00\rangle \equiv\left[\sqrt{m_{q}^{2}+k^{2}}+\sqrt{m_{\bar{q}}^{2}+k^{2}}+V_{q \bar{q}}\right] w^{P S}\left(k^{2}\right)|00\rangle=M_{P S} w^{P S}\left(k^{2}\right)|00\rangle
$$

where $M_{P S}$ is the mass of the $P S$ meson and $V_{q \bar{q}}$ the $G I$ effective $q \bar{q}$ potential, composed by a $O G E$ term (dominant at short separations) and a linear-confining term (dominant at large separations). In what follows, three different forms of $w^{P S}$ will be considered and labeled as $w_{(G I)}^{P S}, w_{(\text {conf })}^{P S}$ and $w_{(H O)}^{P S}$. The first two correspond to the solutions of Eq. (2) obtained using for $V_{q \bar{q}}$ the full GI interaction and only its linear-confining term, respectively. The third one is a simple gaussian-like ansatz $w_{(H O)}^{P S} \propto e^{-k^{2} /\left(2 \beta_{P S}^{2}\right)}$, where the harmonic oscillator $(H O)$ parameter $\beta_{P S}$ has been fixed by requiring that the average value of $k_{\perp}^{2}$ calculated with $w_{(\text {conf })}^{P S}$ and $w_{(H O)}^{P S}$ is the same. In this way $w_{(\text {conf })}^{P S}$ and $w_{(H O)}^{P S}$ correspond to a linear- and quadraticconfining potential with the same scale, respectively. In case of the $D\left(D_{s}\right), B\left(B_{s}\right)$ and $T$ $\left(T_{s}\right)$ mesons, the value of $\beta_{P S}\left(=\sqrt{<k_{\perp}^{2}>_{\text {conf }}}\right)$ is: $\beta_{D\left(D_{s}\right)}=0.386(0.408)(\mathrm{GeV} / \mathrm{c}), \beta_{B\left(B_{s}\right)}=$ $0.417(0.445)(\mathrm{GeV} / \mathrm{c})$ and $\beta_{T\left(T_{s}\right)}=0.440(0.472)(\mathrm{GeV} / \mathrm{c})$. For the above-mentioned mesons Eq. (2) has been solved by expanding the wave function onto a (truncated) set of $\mathrm{HO}$ basis states and by applying the Raleigh-Ritz variational principle to the coefficients of the expansion. It has been checked that the convergence for all the quantities considered in this letter is reached completely when all the basis states up to $40 \mathrm{HO}$ excitation quanta are included in the expansion. The $C Q$ 's masses are fixed at the values adopted in [9] to 
reproduce meson mass spectra, namely: $m_{u}=m_{d}=0.220 \mathrm{GeV}, m_{s}=0.419 \mathrm{GeV}, m_{c}=$ $1.628 \mathrm{GeV}$ and $m_{b}=4.977 \mathrm{GeV}$. As to the $t$-quark mass, the value $m_{t}=160 \mathrm{GeV}$ is considered. It should be pointed out that the "inertia" parameter $\bar{\Lambda} \equiv M_{P S}-m_{Q}$, often introduced in the Heavy Quark Effective Theory [4], calculated using the GI interaction for the $T\left(T_{s}\right)$ meson, is found to be $0.38(0.46) \mathrm{GeV}$, in agreement with the expectations of recent lattice $Q C D$ simulations [16].

The $C Q$ momentum distribution $\left|w^{P S}\left(k^{2}\right)\right|^{2}$, calculated for the $D, B$ and $T$ mesons using $w_{(\text {conf })}^{P S}$ and $w_{(G I)}^{P S}$, is shown in Fig. 1(a), while the comparison among $\left|w_{(H O)}^{B}\right|^{2},\left|w_{(\text {conf })}^{B}\right|^{2}$ and $\left|w_{(G I)}^{B}\right|^{2}$ is illustrated in Fig. 1(b), where the gaussian-like wave function of Ref. [5] is also reported. It can be seen that: i) the $O G E$ part of the $G I$ effective interaction generates a huge amount of high-momentum components at $k>1(\mathrm{GeV} / \mathrm{c})$ (cf. [10]); ii) the behaviours of $w_{(H O)}^{P S}$ and $w_{(\text {conf })}^{P S}$ are very similar at low momenta $(k<1(G e V / c))$; iii) the wave functions $w_{(H O)}^{P S}$ do not differ appreciably from the wave functions adopted in [5]. Similar results hold as well for the $C Q$ momentum distribution in $D_{s}, B_{s}$ and $T_{s}$ mesons. It should be pointed out that the $O G E$ term of the $G I$ interaction generates high-momentum components also in the wave function of light mesons and baryons, and sharply affects their e.m. form factors both at low and high $q^{2}$ (see [10, 11, 12]).

Within the light-front formalism any e.m. form factor can be evaluated in terms of the matrix elements of the plus component of the e.m. current operator [14. We stress again that for space-like values of $q^{2}$ a reference frame where $q^{+}=0$ can be chosen in order to suppress the contribution of the pair creation from the vacuum [13]. In what follows the heavy quark $Q$ is assumed to be point-like and a one-body approximation for the e.m. current is adopted. Therefore, the $I W$ function is simply related to the matrix element of the "good" component of the e.m. heavy-quark current, $\bar{Q} \gamma^{+} Q$, by

$$
\xi^{(I W)}(\omega)=\lim _{m_{Q} \rightarrow \infty} H_{P S}^{Q}\left(q^{2}\right)=\lim _{m_{Q} \rightarrow \infty}\left\langle P^{\prime}\left|\bar{Q} \gamma^{+} Q\right| P\right\rangle / 2 P^{+}
$$

where $q^{2}=2 M_{P S}^{2}(1-\omega)$. The explicit expression for $H_{P S}^{Q}\left(q^{2}\right)$ is given by (cf. [10)

$$
H_{P S}^{Q}\left(q^{2}\right)=\int d \vec{k}_{\perp} d \xi \sqrt{J\left(\vec{k}_{\perp}, \xi\right) J\left(\vec{k}_{\perp}, \xi\right)} w^{P S}\left(k^{2}\right) w^{P S}\left({k^{\prime}}^{2}\right) R\left(\vec{k}_{\perp}, \vec{k}_{\perp}, \xi\right)
$$

with $\overrightarrow{k_{\perp}^{\prime}}=\vec{k}_{\perp}+(1-\xi) \vec{q}_{\perp}, M_{0}^{\prime 2}=\frac{k_{\perp}^{\prime 2}+m_{Q}^{2}}{\xi}+\frac{{k^{\prime}}_{\perp}^{2}+m_{s p}^{2}}{(1-\xi)}, k_{n}^{\prime}=\left(\xi-\frac{1}{2}\right) M_{0}^{\prime}+\frac{m_{s p}^{2}-m_{Q}^{2}}{2 M_{0}^{\prime}}$ and $\left|\vec{q}_{\perp}\right|^{2}=-q^{2}$. In Eq. (ब) $R$ is the contribution of the Melosh rotations and reads as

$$
R\left(\vec{k}_{\perp},{\overrightarrow{k^{\prime}}}_{\perp}, \xi\right)=\frac{\xi(1-\xi)\left[M_{0}^{2}-\left(m_{Q}-m_{s p}\right)^{2}\right]+\vec{k}_{\perp} \cdot\left({\overrightarrow{k^{\prime}}}_{\perp}-\vec{k}_{\perp}\right)}{\xi(1-\xi) \sqrt{M_{0}^{2}-\left(m_{Q}-m_{s p}\right)^{2}} \sqrt{M_{0}^{\prime 2}-\left(m_{Q}-m_{s p}\right)^{2}}}
$$

where $m_{s p}$ is the spectator-quark mass. Since the radial wave function is normalized as $\int_{0}^{\infty} d k k^{2}\left|w^{P S}\left(k^{2}\right)\right|^{2}=1$, one has $H_{P S}^{Q}(\omega=1)=1$ both at finite and infinite heavy-quark masses.

The values of the slope of the body form factor $H_{P S}^{Q}$ at the zero-recoil point, $\rho_{Q}^{2} \equiv$ $-\left[d H_{P S}^{Q}(\omega) / d \omega\right]_{\omega=1}$, calculated using $w_{(H O)}^{P S}, w_{(\text {conf })}^{P S}$ and $w_{(G I)}^{P S}$, are reported in Table 1 (2) for 
the $D\left(D_{s}\right), B\left(B_{s}\right)$ and $T\left(T_{s}\right)$ mesons. The results of the extrapolation to infinite $P S$ meson masses, obtained through a simple quadratic polynomial in terms of $1 / M_{P S}$, are also shown and compared with the predictions of the $I S G W$ [5] and the relativistic flux tube $(R F T)$ [17] models, as well as with recent $Q C D$ sum rule [18, 19] and lattice $Q C D$ results [20, 21]. It can be seen that: i) in the limit of infinite heavy-quark masses the choice of the heavy-meson wave function affects the slope only by $\sim 10 \div 25 \%$; ii) the asymptotic value is reached from below and, in particular, the slope of $H_{P S=D\left(D_{s}\right)}^{Q=c}$ is $\sim 10 \div 30 \%$ lower than its asymptotic value; iii) the effects of the Melosh rotations of the $C Q$ spins (which are necessary for ensuring the correct transformation properties of the meson wave function under kinematical light-front boosts) are relevant both at finite and infinite heavy-quark masses; iv) the slope of the $I W$ form factor, calculated at $m_{s p}=0.220(0.419) \mathrm{GeV}$, is $\rho^{2}=1.03$ (1.14), i.e. it is larger than the $I S G W$ prediction $\rho^{2} \sim 0.6(0.8)$ [5] and in agreement with both $Q C D$ sum rule results [18, 19] and lattice $Q C D$ simulations [20, 21] as well as with the prediction of Ref. [17]. The $\omega$-dependence of the body form factor $H_{P S}^{Q}$, calculated for the $D, B$ and $T$ mesons using $w_{(\text {conf })}^{P S}$ and $w_{(G I)}^{P S}$, is illustrated in Fig. 2. The results obtained with $w_{(H O)}^{P S}$ turn out to be very similar to the ones calculated using $w_{(\text {conf })}^{P S}$. From Fig. 2 it can be seen that, in a wide range of values of the recoil, the Melosh composition of the $C Q$ spins remarkably affects the calculations performed both with and without the high-momentum components generated in the heavy-meson wave function by the $O G E$ interaction. Moreover, the results obtained at the $T$-meson mass $(\sim 160 \mathrm{GeV})$ can be considered representative of the limit of infinite heavy-quark masses. Therefore, the $I W$ form factor $\xi^{(I W)}(\omega)$, obtained through Eq. (4) using $w_{(H O)}^{T\left(T_{s}\right)}, w_{(\text {conf })}^{T\left(T_{s}\right)}$ and $w_{(G I)}^{T\left(T_{s}\right)}$, is shown in Fig. 3. It can be seen that in a wide range of values of the recoil the calculated $I W$ form factor exhibits a moderate dependence upon the choice of the heavy-meson wave function; in particular, it is slightly affected by the high-momentum components present in $w_{(G I)}^{P S}$, as well as by the detailed form of the confining interaction. Therefore, the $I W$ form factor is found to be mainly governed by the effects of the confinement scale. Such a feature can be ascribed to an end-point mechanism, namely to the fact that the heavy-meson wave function is sharply peaked at $\xi \sim 1-m_{s p} / m_{Q}$, which implies that in Eq. (四) $\left|{\overrightarrow{k^{\prime}}}_{\perp}-\vec{k}_{\perp}\right| \sim m_{s p} \sqrt{2(\omega-1)}$, enhancing the contribution due to the low- $k_{\perp}\left(k_{\perp}^{\prime}\right)$ components of the heavy-meson wave function. It is only for $\omega>10(5)$ that the high-momentum components present in $w_{(G I)}^{P S}$ can remarkably affect $\xi^{(I W)}(\omega)$ at $m_{s p}=0.220(0.419) G e V$ 目.

Finally, in Fig. 4 our prediction for $\xi^{(I W)}(\omega)$, calculated at $m_{s p}=0.220 \mathrm{GeV}$, is compared with the results obtained within various approaches, namely the $I S G W$ prediction $\xi^{(I W)}(\omega)=e^{-\rho^{2}(\omega-1)}$ with $\rho^{2}=0.60$ [5], the result of the RFT model [17] and the calculation of Ref. [22], based on a $Q C D$ sum rule analysis at next-to-leading order in renormalization-group improved perturbation theory. It can be seen that our result, as well as the relativistic calculation of Ref. [17], differ only slightly from the prediction of Ref. [22], whereas the $I S G W$ model [5], characterized by an approximate treatment of the relativistic

\footnotetext{
${ }^{b}$ Note also from Fig. 3 that, according to the above-mentioned end-point mechanism, a little bit higher sensitivity to high-momentum components is found for $\omega<4$ when $m_{s p}=0.419 \mathrm{GeV}$.
} 
effects, predicts higher values for $\xi^{(I W)}(\omega)$.

To sum up, the space-like elastic form factor of heavy-light pseudoscalar mesons has been investigated within a light-front constituent quark model in order to evaluate the IsgurWise function. It has been shown that in the space-like sector the Melosh composition of the constituent quark spins plays a relevant role. Moreover, it turns out that in a wide range of values of the recoil the calculated Isgur-Wise form factor exhibits a moderate dependence upon the choice of the heavy-meson wave function; in particular, $\xi^{(I W)}(\omega)$ is slightly affected by the high-momentum components generated by the one-gluon-exchange term of the effective $q \bar{q}$ interaction, as well as by the detailed form of the confining potential. This fact suggests that the $I W$ form factor is mainly governed by the effects of the confinement scale. The slope of $\xi^{(I W)}(\omega)$, calculated at $m_{s p}=0.220(0.419) \mathrm{GeV}$, is $\rho^{2}=1.03$ (1.14), i.e. it is larger than the $I S G W$ prediction $\rho^{2} \sim 0.6(0.8)$ [5] and in agreement with both $Q C D$ sum rule [18, 19] and lattice $Q C D$ results [20, 21].

The application of our light-front constituent-quark model to the time-like sector (i.e., in the kinematical regions pertaining to the weak decays of heavy mesons), as well as the comparison of the elastic and transition form factors of heavy-light pseudoscalar mesons in the limit of infinite heavy-quark masses, are in progress and will be reported elsewhere [23].

Acknowledgment. The author gratefully acknowledges Fabio Cardarelli for supplying him with the numerical solutions of Eq. (2).

\section{References}

[1] N. Cabibbo: Phys. Rev. Lett. 10 (1963) 1531. M. Kobayashi and T. Maskawa: Prog. Theor. Phys. 39 (1973) 653.

[2] N. Isgur and M.B. Wise: Phys. Lett. 232B (1989) 113; Phys. Lett. 237B (1990) 527.

[3] D. Politzer and M.B. Wise: Phys. Lett. 208B (1988) 504. M.B. Voloshin and M.A. Shifman: Sov. J. Nucl. Phys. 47 (1988) 511. H. Georgi: Phys. Lett. 240B (1990) 447.

[4] see for a review: M. Neubert: Phys. Rep. 245 (1994) 259.

[5] D. Scora and N. Isgur: Phys. Rev. D52 (1995) 2783. N. Isgur: Phys. Rev. D43 (1991) 810. N. Isgur, D. Scora, B. Grinstein and M.B. Wise: Phys. Rev. D39 (1989) 799.

[6] M. Wirbel, B. Stech and M. Bauer: Z. Phys. C29 (1985) 237. M. Bauer, B. Stech and M. Wirbel: Z. Phys. C34 (1987) 103. M. Bauer and M. Wirbel: Z. Phys. C42 (1989) 671. 
[7] F.E. Close and A. Wambach: Nucl. Phys. B412 (1994) 169; Phys. Lett. B349 (1995) 207.

[8] H.J. Melosh: Phys. Rev. D9 (1974) 1095.

[9] S. Godfrey and N. Isgur: Phys. Rev. D32 (1985) 185.

[10] F. Cardarelli, I.L. Grach, I.M. Narodetskii, E. Pace, G. Salmé and S. Simula: Phys. Lett. 332B (1994) 1; nucl-th 9509038, to appear in Phys. Rev. D, brief report.

[11] F. Cardarelli, I.L. Grach, I.M. Narodetskii, G. Salmé and S. Simula: Phys. Lett. 349B (1995) 393; Phys. Lett. 359B (1995) 1; Few-Body Systems Suppl. 9 (1995) 267.

[12] F. Cardarelli, E. Pace, G. Salmè and S. Simula: Phys. Lett. 357B (1995) 267; Few-Body Systems Suppl. 8 (1995) 345; preprint INFN-ISS 95/12, nucl-th 9509033, to appear in Phys. Lett. B.

[13] G.P. Lepage and S.J. Brodsky: Phys. Rev. D22 (1980) 2157. L.L. Frankfurt and M.I. Strikman: Nucl. Phys. B148 (1979) 107. M. Sawicki: Phys. Rev. D46 (1992) 474.

[14] For a review see B.D. Keister and W.N. Polyzou: Adv. Nucl. Phys. 20 (1991) 225 and F. Coester: Progress in Part. and Nucl. Phys. 29 (1992) 1.

[15] W. Jaus: Phys. Rev. D44 (1991) 2851 and references therein quoted.

[16] C.T.H. Davies et al.: Phys. Rev. Lett. 73 (1994) 2654.

[17] M.G. Olsson and S. Veseli: Phys. Rev. D51 (1995) 2224.

[18] B. Block and M. Shifman: Phys. Rev. D47 (1993) 2949.

[19] S. Narison: Phys. Lett. 325B (1994) 197.

[20] UKQCD collaboration: Nucl. Phys. (Proc. Suppl.) B42 (1995) 2224.

[21] C.W. Bernard, Y. Shen and A. Soni: Nucl. Phys. (Proc. Suppl.) B34 (1994) 483.

[22] M. Neubert: Phys. Rev. D47 (1993) 4063.

[23] S. Simula et al.: to be published. 


\section{Table Captions}

Table 1. The slope $\rho_{Q}^{2} \equiv-\left[d H_{P S}^{Q}(\omega) / d \omega\right]_{\omega=1}$ of the body form factor $H_{P S}^{Q}$ (Eq. (4) with $q^{2}=2 M_{P S}^{2}(1-\omega)$ ), calculated for the $D, B$ and $T$ mesons using different radial wave function $w^{P S}\left(k^{2}\right): w_{(H O)}^{P S} \propto e^{-k^{2} / 2 \beta_{q \bar{q}}^{2}}, w_{(c o n f)}^{P S}$ and $w_{(G I)}^{P S}$. The first two correspond to a quadratic- and linear-confining potential with the same scale, respectively (see text). The wave function $w_{(G I)}^{P S}$ is the solution of Eq. (2) using for $V_{q \bar{q}}$ the full $G I$ effective interaction [9]. The result obtained by neglecting the effects of the Melosh rotations of the $C Q$ spins, i.e. by assuming $R=1$ in Eq. (11), is also reported. The last column corresponds to the asymptotic limit of infinite heavy-quark masses, calculated through a quadratic polynomial extrapolation in terms of $1 / M_{P S}$ of the results obtained at finite values of $M_{P S}$. The mass of the spectator-quark is $m_{s p}=0.220 \mathrm{GeV}$. For comparison, the predictions of the $I S G W$ [5] and RFT [17 models, as well as recent results from $Q C D$ sum rule $(Q C D-S R)$ 18, 19 and lattice $Q C D$ simulations [20], are shown.

Table 2. The same as in Table 1 , but for the $D_{s}, B_{s}$ and $T_{s}$ mesons, using a spectatorquark mass of $0.419 \mathrm{GeV}$. For comparison, the predictions of the $I S G W$ model [5] and the results of recent lattice $Q C D$ calculations [20, 21] are reported. 


\section{Figure Captions}

Fig. 1. (a) The $C Q$ momentum distribution $\left|w^{P S}\left(k^{2}\right)\right|^{2}$ versus the internal momentum $k$. The dot-dashed, dashed and solid lines correspond to the $D, B$ and $T$ mesons, respectively. Thin and thick lines correspond to $w_{(G I)}^{P S}$ and $w_{(\text {conf })}^{P S}$, which are solutions of Eq. (2) obtained using for $V_{q \bar{q}}$ the full GI effective interaction [9] and only its linear-confining term, respectively. (b) The $C Q$ momentum distribution in the B-meson. The dotted, dashed and solid lines correspond to $w_{(\text {conf })}^{B}, w_{(H O)}^{B} \propto e^{-k^{2} /\left(2 \beta_{B}^{2}\right)}\left(\right.$ with $\beta_{B}=0.417(G e V / c)$ ) and $w_{(G I)}^{B}$, respectively. The dot-dashed line corresponds to the gaussian-like wave function of Ref. [5].

Fig. 2. (a) Body form factor $H_{P S}^{Q}(\omega)$ (Eq. (4) with $q^{2}=2 M_{P S}^{2}(1-\omega)$ ) versus $\omega$, calculated with $w_{(\text {conf })}^{P S}\left(k^{2}\right)$ which corresponds to the solution of Eq. (2) using for $V_{q \bar{q}}$ the linear-confining term of the $G I$ effective interaction [9]. The dotted, dashed and solid lines correspond to the $D, B$ and $T$ mesons, respectively. The dot-dashed line is the result obtained for the $T$ meson by neglecting the effects of the Melosh rotations of the $C Q$ spins, i.e. by assuming $R=1$ in Eq. (乘). (b) The same as in (a) but using $w_{(G I)}^{P S}\left(k^{2}\right)$ which corresponds to the solution of Eq. (2) with the full GI effective interaction.

Fig. 3. The $I W$ form factor $\xi^{(I W)}(\omega)$ versus $\omega$ for a spectator-quark mass of $0.220 \mathrm{GeV}$ (a) and $0.419 \mathrm{GeV}$ (b). The dashed, dotted and solid lines correspond to the results obtained using in Eq. (四) $w_{(H O)}^{T\left(T_{s}\right)}, w_{(\text {conf })}^{T\left(T_{s}\right)}$ and $w_{(G I)}^{T\left(T_{s}\right)}$, respectively. The dot-dashed line is the result obtained for the $T\left(T_{s}\right)$ meson neglecting the effects of the Melosh rotations of the $C Q$ spins (i.e., assuming $R=1$ in Eq. (4)).

Fig. 4. Comparison of the $I W$ form factor $\xi^{(I W)}(\omega)$ calculated in various approaches. The solid line is our result obtained using in Eq. (四) $w_{(G I)}^{T}\left(k^{2}\right)$ and a spectator-quark mass of $0.220 \mathrm{GeV}$. The dot-dashed line corresponds to the $I S G W$ prediction $\xi^{(I W)}(\omega)=e^{-\rho^{2}(\omega-1)}$ with $\rho^{2}=0.60$ [5]. The dashed line is the result of the RFT model [17] and the dotted line is the prediction of Ref. [22], based on a $Q C D$ sum rule analysis at next-to-leading order in renormalization-group improved perturbation theory. 
TABLE 1

\begin{tabular}{||c||c|c|c||c||}
\hline$w^{P S}\left(k^{2}\right)$ & D-meson & B-meson & T-meson & $\infty$ \\
\hline$H O$ & 1.01 & 1.16 & 1.25 & 1.26 \\
\hline conf. & 0.94 & 1.06 & 1.12 & 1.13 \\
\hline$G I$ & 0.68 & 0.88 & 1.02 & 1.03 \\
\hline$G I(R=1)$ & 0.39 & 0.54 & 0.69 & 0.70 \\
\hline \hline$I S G W[5]$ & & & & $0.5 \div 0.6$ \\
\hline$R F T[17]$ & & & & $0.93 \pm 0.04$ \\
\hline$Q C D-S R \llbracket 18]$ & & & & $0.70 \pm 0.25$ \\
\hline$Q C D-S R[19]$ & & & & $1.00 \pm 0.02$ \\
\hline$U K Q C D[20]$ & & & & $0.9_{-.3-.2}^{+.2+.4}$ \\
\hline
\end{tabular}

TABLE 2

\begin{tabular}{||c||c|c|c||c||}
\hline$w^{P S}\left(k^{2}\right)$ & $D_{s}-$ meson & $B_{s}-$ meson & $T_{s}-$ meson & $\infty$ \\
\hline$H O$ & 1.27 & 1.40 & 1.48 & 1.48 \\
\hline conf. & 1.23 & 1.32 & 1.37 & 1.37 \\
\hline$G I$ & 0.77 & 0.98 & 1.14 & 1.14 \\
\hline$G I(R=1)$ & 0.43 & 0.65 & 0.84 & 0.84 \\
\hline \hline$I S G W[5]$ & & & & $0.7 \div 0.8$ \\
\hline$U K Q C D[20]$ & & & & $1.2_{-.2+.1}^{+.2+.2}$ \\
\hline$B S S[21]$ & & & & $1.24 \pm 0.26 \pm 0.36$ \\
\hline
\end{tabular}




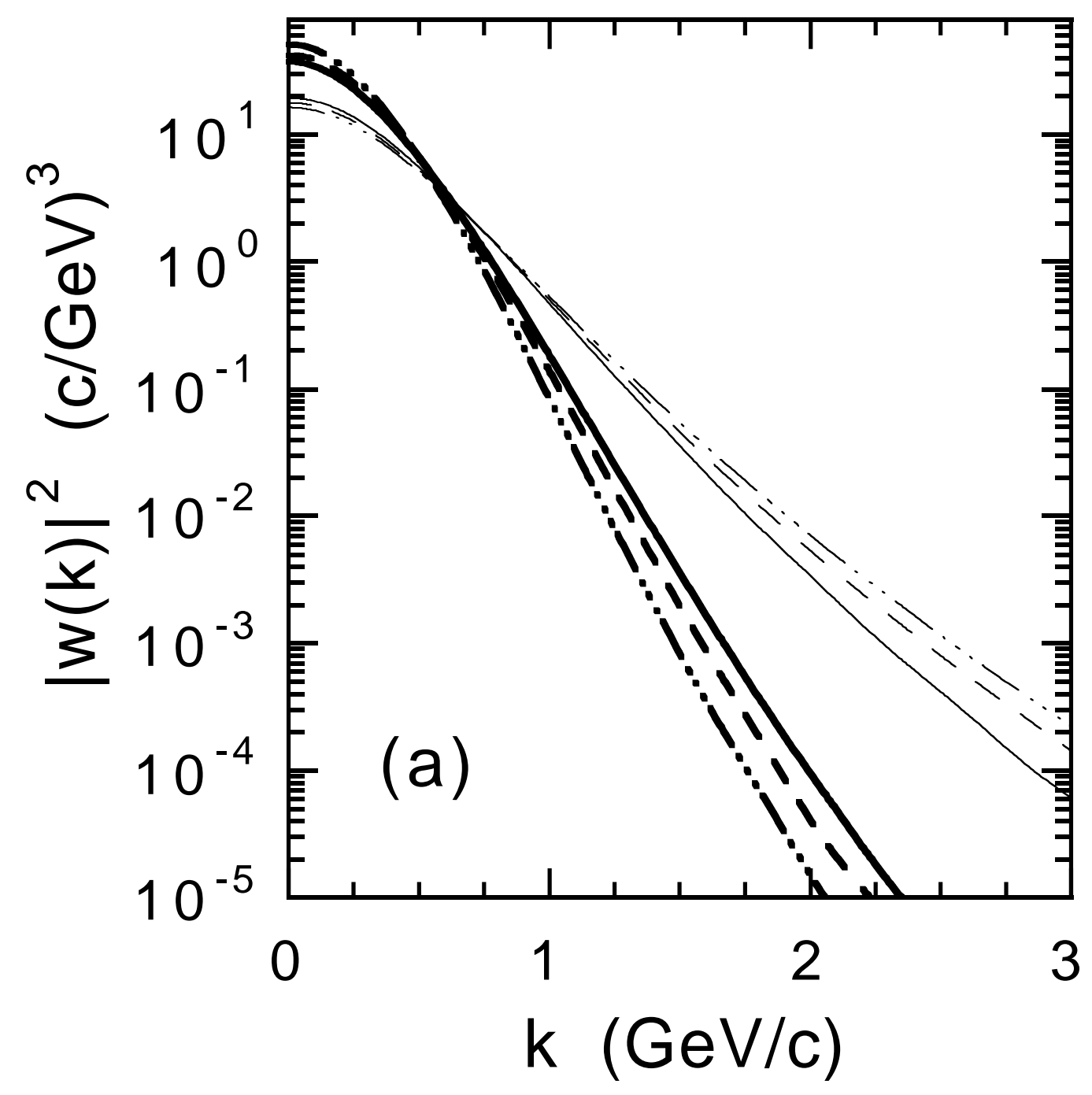

S. Simula, Phys. Lett. B: fig. 1(a). 


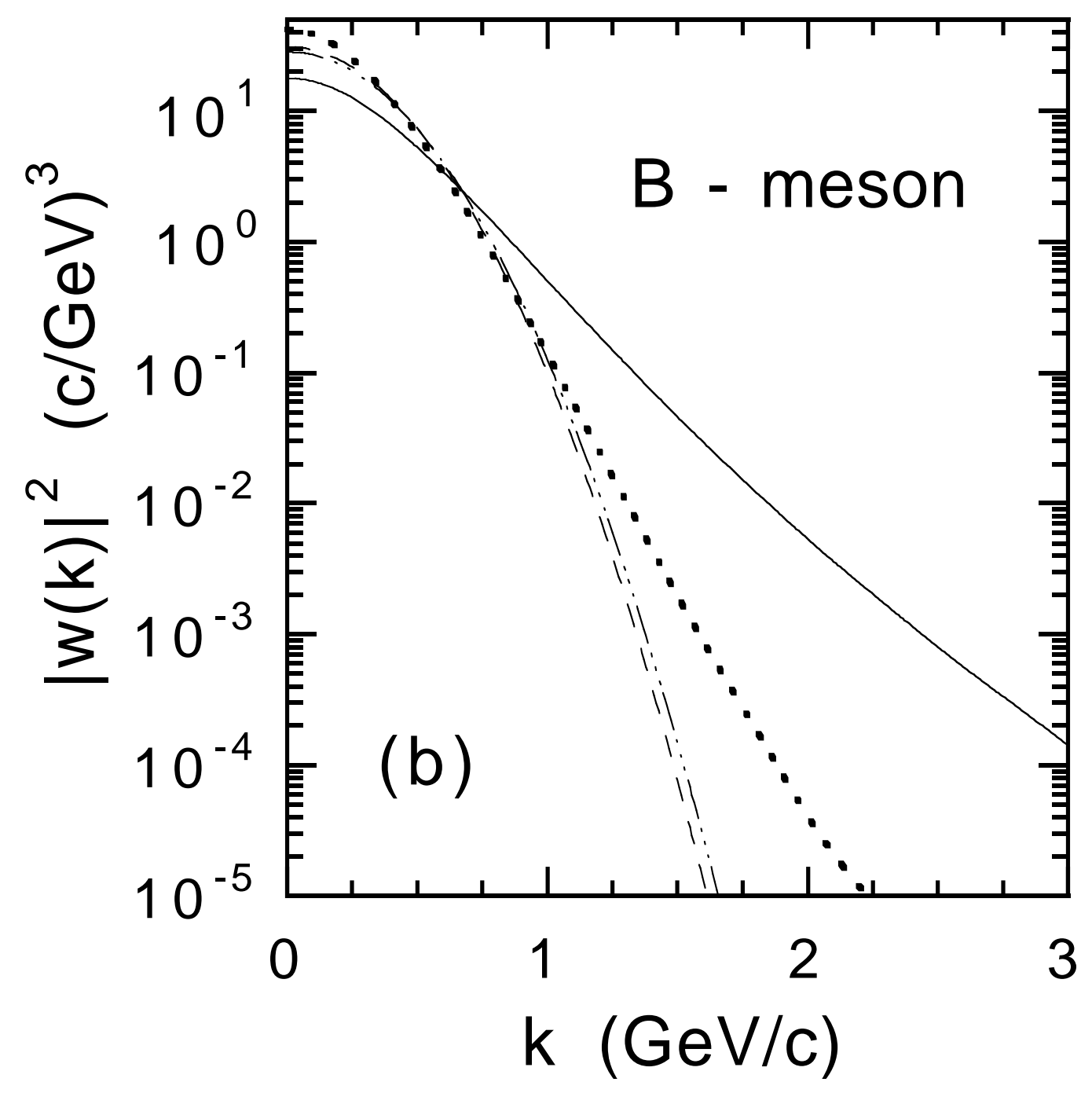

S. Simula, Phys. Lett. B: fig. 1(b). 


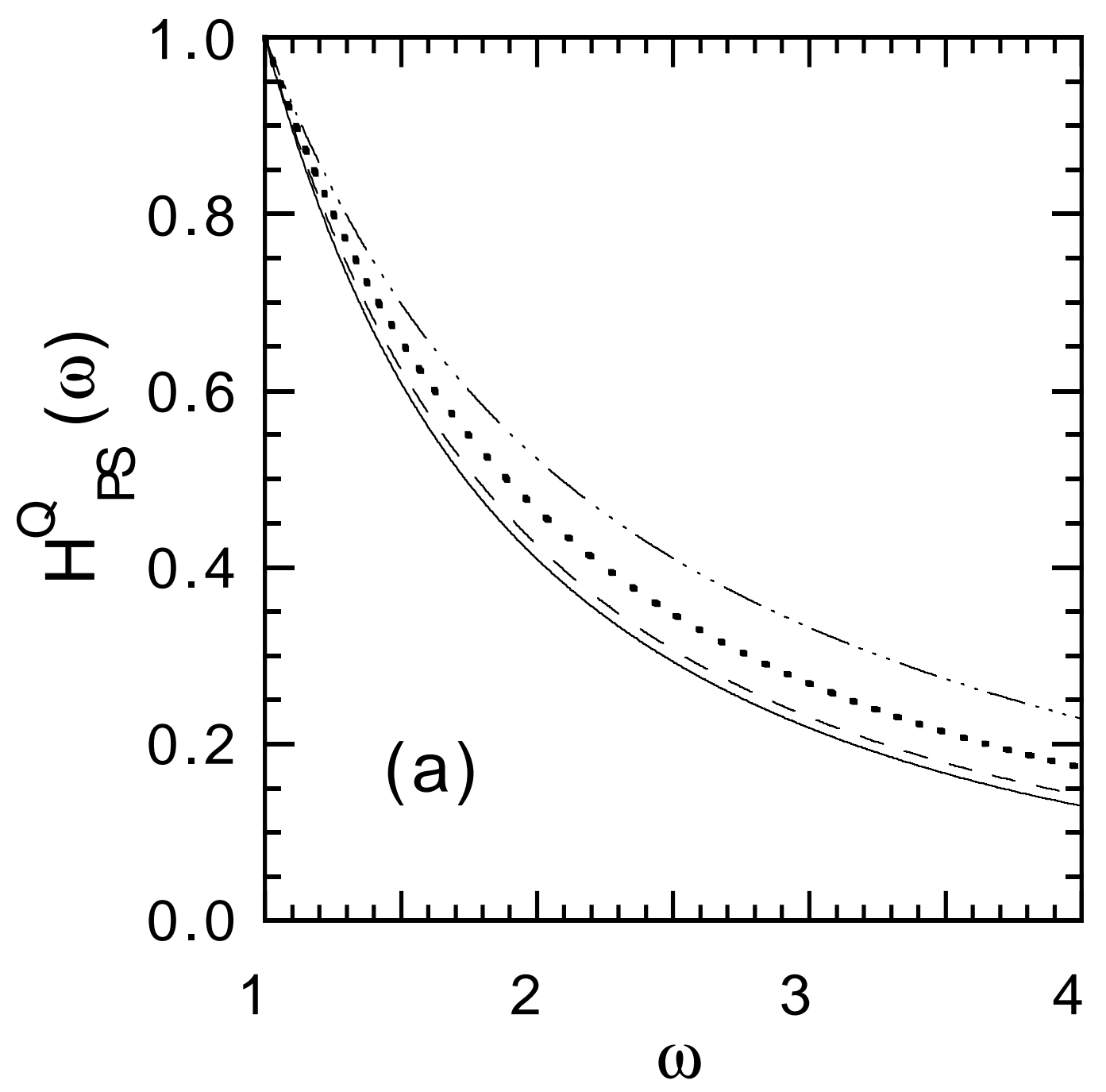

S. Simula, Phys. Lett. B: fig. 2(a). 


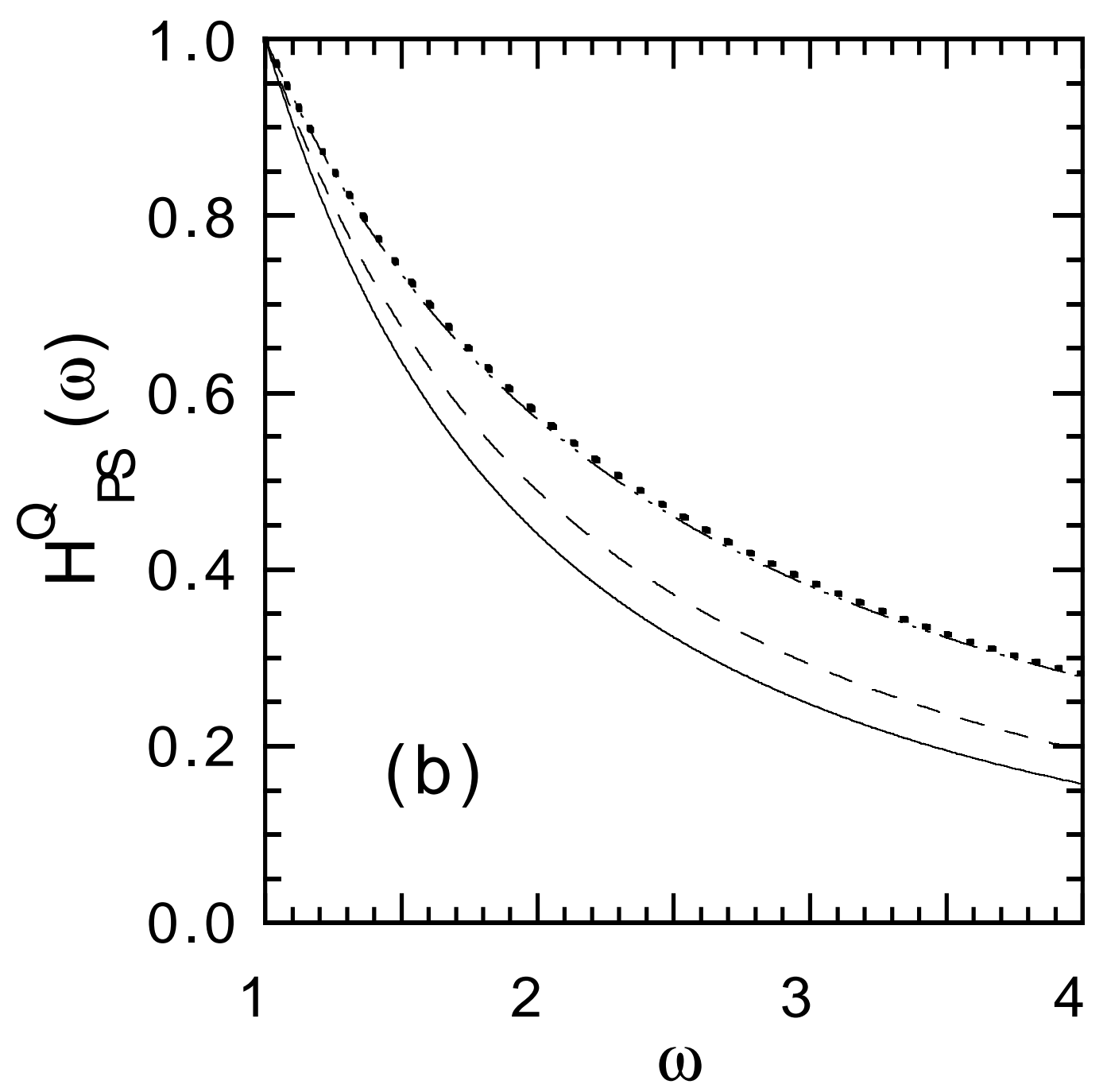

S. Simula, Phys. Lett. B: fig. 2(b). 


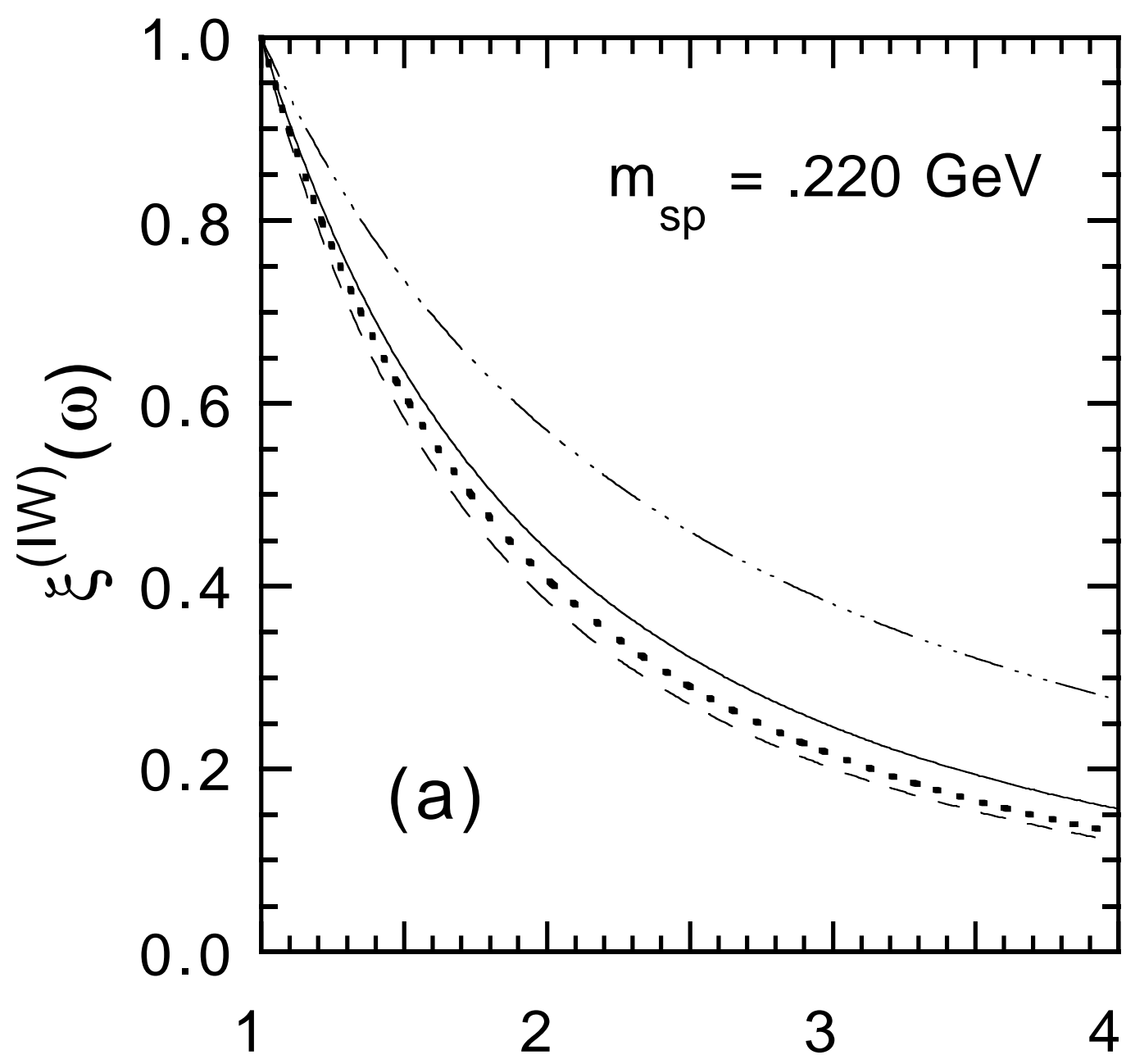

$\omega$

S. Simula, Phys. Lett. B: fig. 3(a). 


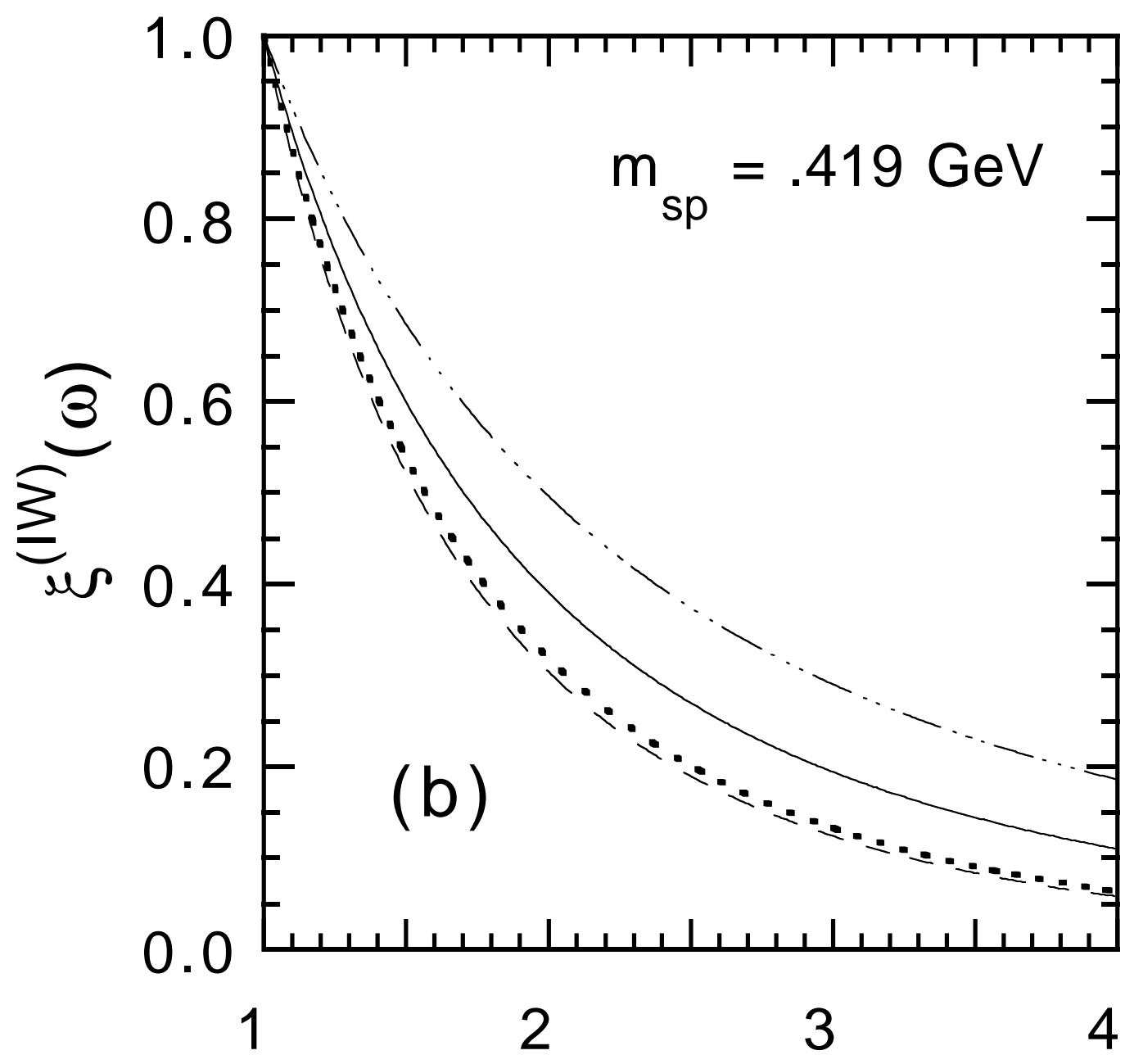

$\omega$

S. Simula, Phys. Lett. B: fig. 3(b). 


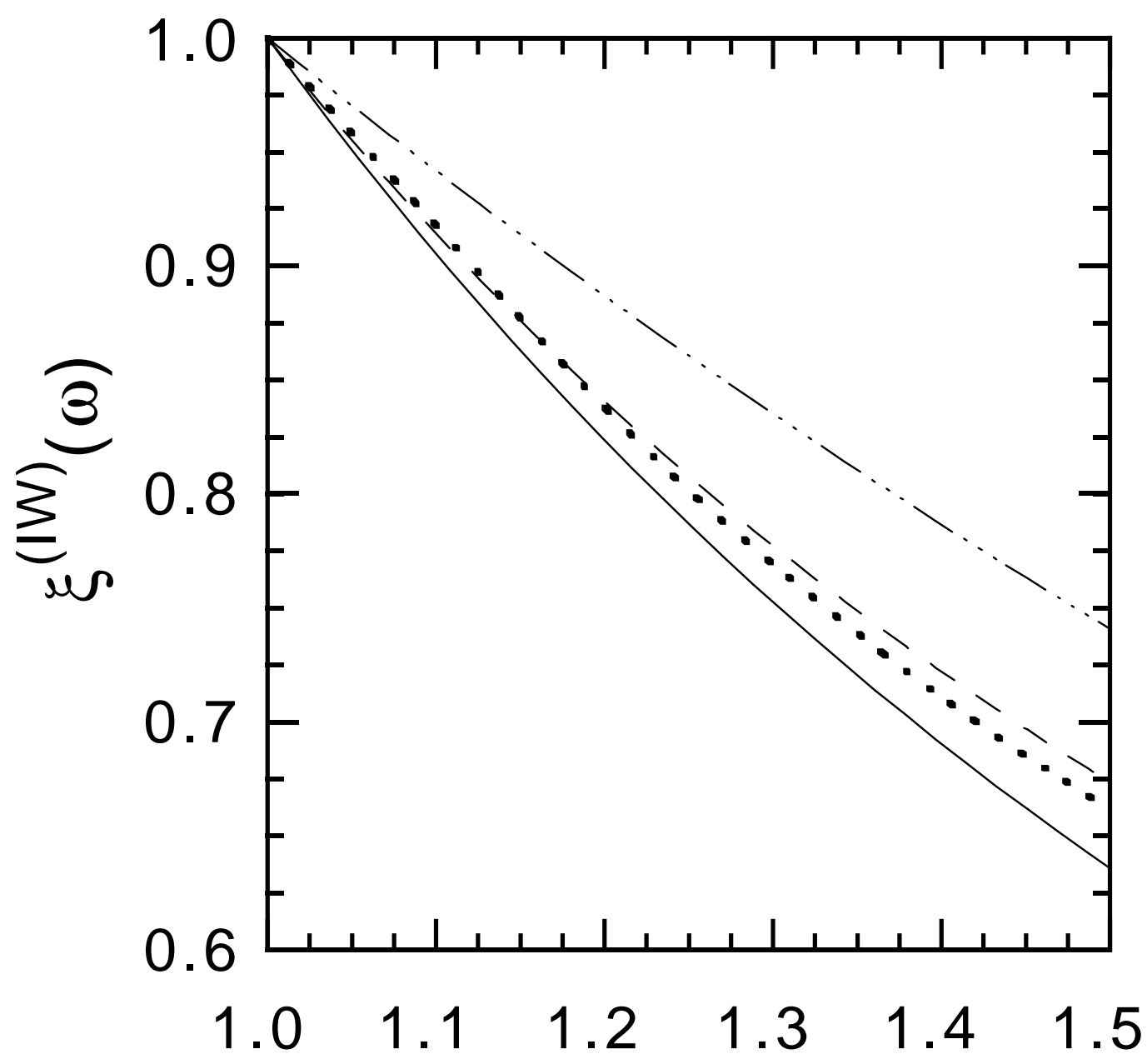

$\omega$

S. Simula, Phys. Lett. B: fig. 4. 